\title{
Summary of the Adaptive Command and Control Organizational Design Based on Granular Computing
}

\author{
Jie $\mathrm{Fu}^{1,}$, Xuejun Ren ${ }^{1}$, Jisheng $\mathrm{Zhu}^{1}$ and Xiaohong Wang ${ }^{2}$ \\ ${ }^{1}$ Engineering University of the Chinese people's armed police force, Xi'an 710086, China \\ ${ }^{2}$ The Communication Terminal of Armed Police Headquarters, Beijing 100000, China \\ afujie1007@qq.com
}

\begin{abstract}
This paper introduces the research status of adaptive Command and control (C2) organization, and analyzes the basic design idea of organization adaptive design method based on granular computing. It puts forward a question against the granulating process of platform sets. In addition, it compares the method of adaptability adjustment based on the three-stage approach and the method based on the granular computing, and explains the advantages and disadvantages of the two methods. Finally, it discusses the prospect of future research work.
\end{abstract}

Keywords: Adaptive C2 organization; C2 organizational structure design; Random search algorithm; Adjustment methods of adaptability.

\section{Introduction}

The rapid development of modern science and technology, variability of organization's environment, as well as the complex mission and tasks make the development of organization face new challenges. The rapid development of modern organization is inseparable from the impact and promotion of new technologies. Currently, the development of the organization has already broken the limitations on region, organizational resource and fixed structure, showing the characteristics of distribution, autonomy, flexibility and flat ${ }^{[1]}$.

There are currently two research directions for solving design problems of organizational structure in dynamic and uncertain mission environment ${ }^{[2]}$. One is the adaptive design of organizational structure, and the other is robustness design of organizational structure. Wherein, the adaptability refers to establishing optimal organizational structure under certain mission environment, and reconstructing the organization with certain techniques according to the changes of environment, so as to maintain the smooth operation of the organization ${ }^{[3]}$.

It is difficult for the designer of the organization to obtain real-time and accurate organizational design parameters as the mission environment is complicated and changeable ${ }^{[4]}$. In addition, the occurrence of something unexpected in the execution of mission will also make the original static organizational structure difficult to adapt to the current situation. In order to solve the problem of organizational structure design in dynamic mission environment, optimized organizational structure can be established under certain mission environment, and then carry out dynamic reconfiguration according to the change in the external environment and the process of mission mandate, so as to remain the good performance of the organization all the way. This design approach is called adaptive design of organizational structure.

There are mainly four methods, i.e. organization modeling, testing, organizational design, and organization adaptability adjustment for the research of adaptive $\mathrm{C} 2$ organization. Wherein, the method of $\mathrm{C} 2$ organization modeling mainly includes: modeling based on computational mathematics organization theory, modeling based on Petri, modeling using the method of information theory, modeling based on agent, as well as the modeling based on social network analysis; the design methods of $\mathrm{C} 2$ organization mainly includes: three-stage organizational design method, extended three-stage organizational design method, organizational design method based on packet technology and nested genetic algorithm, and the organizational design method based on granular computing ${ }^{[5]}$; Organization experimental testing methods include: organizational experimental test method based DDD - III, organizational experimental test method based on Extend 
and the organizational full closed loop virtual experimental test method; The adjustment methods of organizational adaptability include: the adjustment method of organizational adaptability based on three-stage approach, the adjustment method of organizational adaptability based on granular computing and the adjustment method of organizational adaptability based on organizational structure stratification ${ }^{[6]}$. This paper mainly discusses the design method based on granular computing.

\section{The Related Concepts of Granular Computing}

The theory of granular computing is mainly applied in adaptive design of organization ${ }^{[7]}$, which specifically includes $\mathrm{C} 2$ organizational structure design based on granular computing, as well as adjustment of organizational adaptation. Structural design of organization is relatively typical for solving complex problems, which mainly relates to platform, tasks, and the design of relationships such as matching, coordination and so on among decision-making entities. The introduction of granular computing approach can reduce the computational complexity. While in the adjustment of organizational adaptability, the adaptability in face of unexpected events (eg: Mission environmental changes, or changes in the internal elements of the organization, etc.) is focused.

\section{The Application of Granular Computing in C2 Organizational Design}

The theory of granular computing is mainly applied in adaptive design of organization, which specifically includes C2 organizational structure design based on granular computing, as well as adjustment of organizational adaptation. Structural design of organization is relatively typical for solving complex problems, which mainly relates to platform, tasks, and the design of relationships such as matching, coordination and so on among decision-making entities. The introduction of granular computing approach can reduce the computational complexity. While in the adjustment of organizational adaptability, the adaptability in face of unexpected events (eg: Mission environmental changes, or changes in the internal elements of the organization, etc.) is focused.

\subsection{C2 Organization Structural Design Based on Granular Computing.}

The optimal design of organization is built on the premise that the matching metric is carried out between organizational structure and mission tasks, therefore, the measurement parameters of organization adaptability need to be determined first. Through the analysis of parameters matched by organizational structure, Xiu Baoxin and others determined the two mainly involved measurement parameters of organization in dealing with mission tasks in Reference 3: external collaboration load $\mathrm{E}(\mathrm{m})$ and the internal working load $\mathrm{I}(\mathrm{m})$ of decision-making entity DM. Both are collectively referred to as the workload of decision-making entities, denoted as $\mathrm{W}(\mathrm{m})$. In the above formula, $\mathrm{W}^{\mathrm{I}}$ and $\mathrm{W}^{\mathrm{E}}$ are the weights。

$$
\mathrm{W}(\mathrm{m})=\mathrm{W}^{\mathrm{I}} \cdot \mathrm{I}(\mathrm{m})+\mathrm{W}^{\mathrm{E}} \cdot \mathrm{E}(\mathrm{m})
$$

The adaptability measurement of organizational structure $\mathrm{O}$ executives mission $\mathrm{M}$ is:

$$
\mathrm{W}_{\mathrm{RMS}}(\mathrm{O}, \mathrm{M})=\sqrt{\frac{1}{m} \Sigma_{\mathrm{w}=1}^{N} W^{2}(\mathrm{~m})}
$$

The purpose of organization adaptive design is to find the transition structure between the current structure and ideal transition structure, making the transition structure adaptive to the new mission environment with small prices.Figure 1 is the flow diagram of design methods.

The design process of $\mathrm{C} 2$ organizational structure based on granular computing is divided into three phases ${ }^{[8]}$.The first stage: to granulate the task set and platform set, and create correspondence between platform granule and task granule. Determine which platforms each decision-making entity has and which tasks it needs to execute; The second stage: intra granular mission planning. Broken down the programming problems with a large number of platforms and tasks into some in dependent sub programming problems with a small number of platforms and missions, determine the specific configuration from platform to task in platform granules and task granules, so as to achieve all the assignation from platform to task, and provide the decomposition method of intra granular 
planning;The third stage: organizational decision-making tree - decision-making stratification. To optimize the allocation of responsibilities of decision-making entity and control collaboration among decision-making entities by specifying communication structure and decision-making stratification. Based on the $\mathrm{C} 2$ organizational structure design which is based on granular computing, this paper designs the granulation for the task set and platform set of the first stage with stochastic search algorithm.

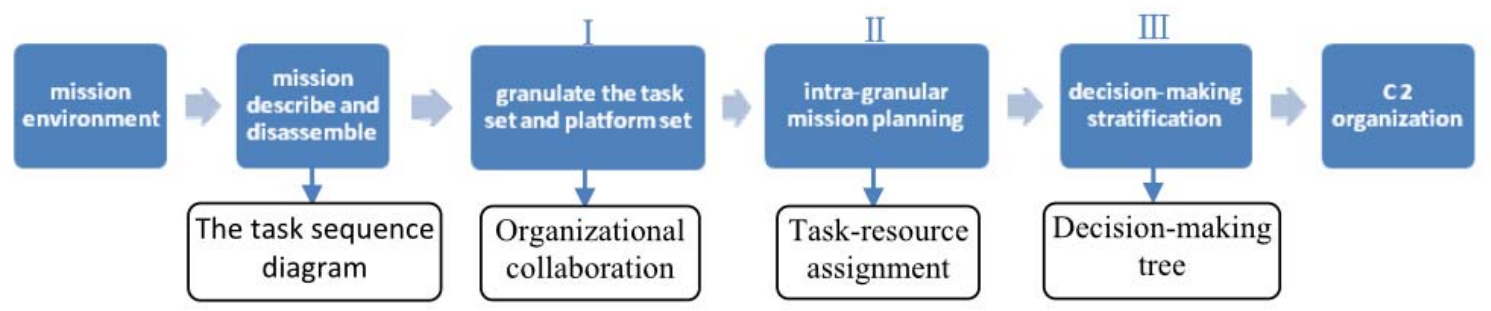

Fig. 1 The flow diagram of $\mathrm{C} 2$ organizational design methods based on granular computing.

Reference 9 presents a random search algorithm (Adaptive Stochastic Search with Hybrid Strategy, HASS) which introduces group discovering and add-in behavior. Compared with traditional genetic algorithm, HASS algorithm integrates discovery policy, add-in strategy and mutation strategy in optimization process to guide optimal individuals and poorer individuals in groups in carrying out global and local searching in the entire search space, which enhances the ability of the algorithm to jump out of local optimum and optimization efficiency, and therefore solves the complex problems of different forms.

HASS algorithm uses the regional hybrid search strategy and variability strategies to improve the optimization performance of the algorithm. First of all, granulate task sets according to ability required by each task in the task set(actually is a particle coverage, because there are multiple decision-making entities to complete a certain task together), and get $\mathrm{M}$ task granules; Take the capability of different decision-making entity as the basis to obtain the correspondence between different task granules and decision-making entities; Carry out granular partition for platform sets with the correspondence between the decision-making entities and the platforms, and obtain $\mathrm{M}$ platform granules; finally get the correspondence between platform granules and task granules , and complete the granulation of platform sets and task sets.

Each platform granule is the aggregation of some function, and each task granule has the similarity of some function. As one platform only belongs to one decision-making entity, one task may be collaboratively completed by a number of decision-making entities ${ }^{[10]}$, therefore, granulating platform sets results in granular partition, and granulating task sets results in granular coverage. Granulation can enable one platform granule to handle one task granule effectively, which is actually to solve the original problem on a new level of granularity, and to simplify the complexity of solving the problem.

(1) Granulation of Task Sets

Divide respectively according to both serial and parallel relations among tasks, and the partition diagram is as shown in Figure 2:

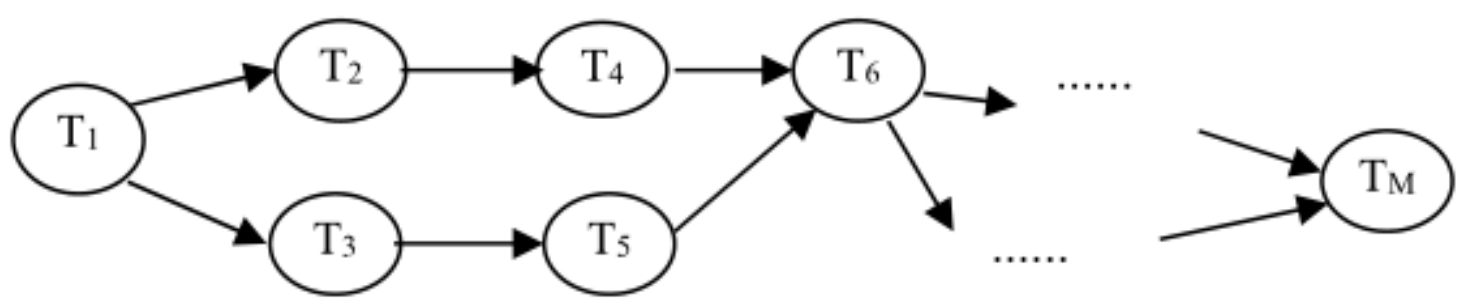

Fig. 2 Schematic Diagram of Task Set Granulation

(2) Granulation of Platform Sets

The process of using HASS algorithm to carry out granular partition for platform sets is as shown in Figure 3:

\subsection{Comparison of Two Adjustment Methods of C2 Organization Adaptability.}

The Design of Organizational Structure Adaptability Based on the Three-stage Approach. 


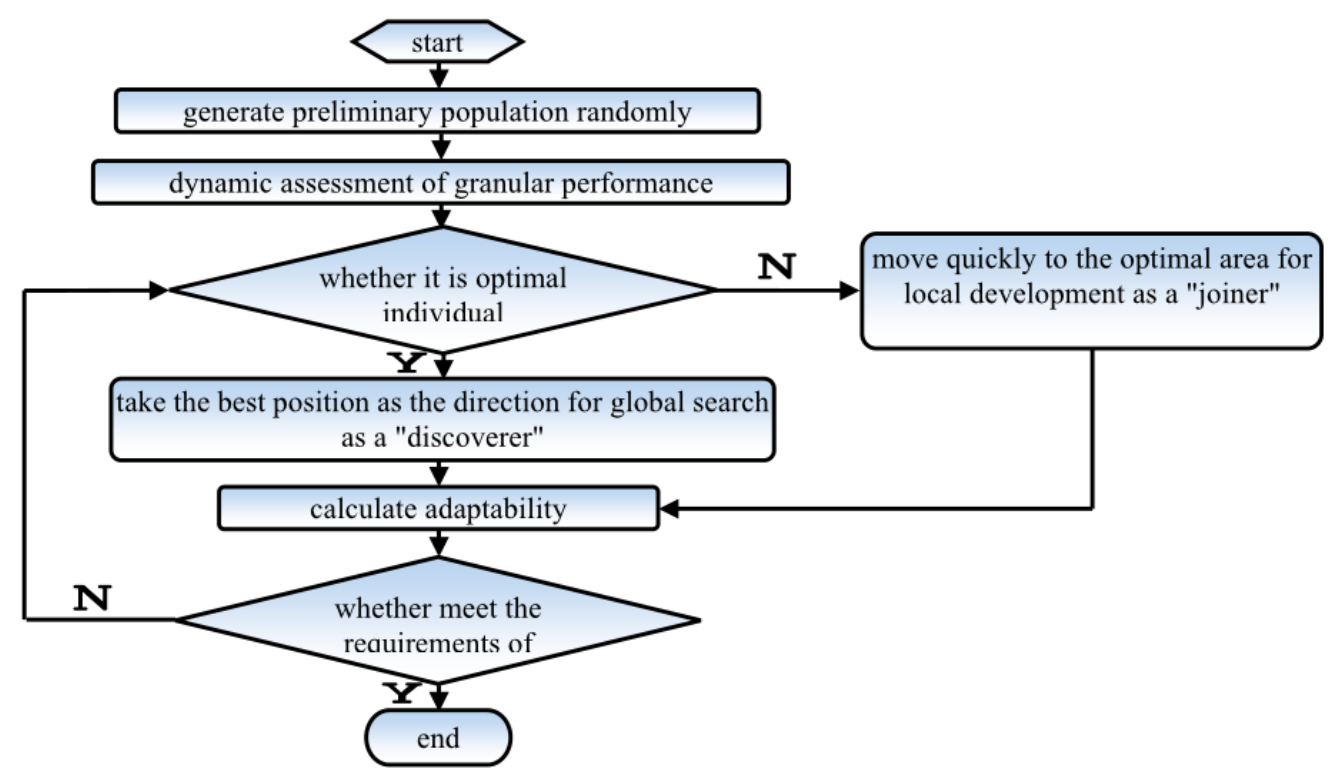

Fig. 3 The process to carry out granular partition for platform sets

Design ideas: the adjustment of organizational structure adaptation is to change the structure into a new one. Two factors need to be taken into consideration in the selection of new organizational structure: the matching degree of mission structure, and the cost rising from structure change. Assess respectively with the performance measurement of organizational structure in finishing mission and the cost function for structure adjustment. Select the optimal structure adaptive to the current mission environment through the above mentioned two indicators from the pre-set alternative structures.

Design process: firstly, divide mission into multiple stages, and calculate the matching degree of mission environment in each stage; according to the path of structure change and the cost rising from structure change of adjacent structure, finally select and determine the structure change routine adaptive to the whole mission execution process: Procedure 1: decompose the mission $\mathrm{M}$ into several small missions according to the execution sequence: $\mathrm{M}_{1}, \mathrm{M}_{2}, \ldots, \mathrm{M}_{\mathrm{n}}$. Design some alternative organizational structures $\mathrm{O}_{1}, \mathrm{O}_{2}, \ldots \mathrm{O}_{\mathrm{k}}$ with the three-stage design method; Process 2: calculate the matching degree $\mathrm{Pij}$ of each $\mathrm{Mj}$ and $\mathrm{Oi}$, and draw the grid of organizational structure changes with these three parameters; Procedure 3: Set the trigger conditions for the organizational structure change, calculate the converting cost of two adjacent structures $\mathrm{O}_{a} \rightarrow \mathrm{Ob}_{b}$. Obtain the optimum path of structure transformation by means of the shortest path algorithm.

Defects of the Design:The organizational three-stage adaptive design is essentially a design method under static conditions. Therefore, if the task is to be handled under dynamic conditions, there would be great difficulties in the organizational design. Firstly, in order to guarantee good integrated organizational adaptive performance, more organizational structures need to be designed so as to be selected in subsequent steps while designing organizational structure with the three-stage method. However, it will definitely increase the complexity to design more organizational structures. Secondly, since the adaptive design is based on the estimation for mission environmental change, when it is faced with random events or sudden changes within the organization which are usually beyond the expectation, it will cause that the existing organizational structure does not match the current mission environment; In addition, as the three-stage design method determines the conversion order of the organizational structure mainly on the basis of the mission sequence after decomposition. The changing mission environment results in the uncertainty of mission sequence. There will be certain risk in the path of structural transformation established on this basis, and its effectiveness has yet to be further discussed.

Comparison of Adjustment Methods for Organizational Adaptability. As organizational design is based on specific mission environment, therefore, different adaptive design methods have their own applicable fields, without absolute advantages or disadvantages. The adjustment methods of organizational adaptability based on three-stage method and granular computing will be compared in the table 1 below. 
Table 1 Caparison of Two Common Adjustment Methods for Organizational Adaptability

\begin{tabular}{|c|c|c|}
\hline Item & Three-stage Method & Granular Computing Method \\
\hline $\begin{array}{l}\text { Applicable Mission } \\
\text { Environment }\end{array}$ & $\begin{array}{l}\text { stable, with few } \\
\text { changes }\end{array}$ & dynamic, uncertain \\
\hline $\begin{array}{c}\text { Construct } \\
\text { Organizational } \\
\text { Structure in Advance }\end{array}$ & needed & not needed \\
\hline $\begin{array}{l}\text { Optimize Time } \\
\text { Domain }\end{array}$ & $\begin{array}{c}\text { Entire mission } \\
\text { execution interval }\end{array}$ & Entire mission execution interval \\
\hline $\begin{array}{l}\text { The relationship } \\
\text { among platforms, } \\
\text { tasks, and } \\
\text { decision-making } \\
\text { entities }\end{array}$ & Fully considered & $\begin{array}{c}\text { Only adjust the command and collaborative } \\
\text { relationships among decision-making entities, and } \\
\text { ownership of platforms and decision making entities. } \\
\text { The design of platform and task assignments } \\
\text { relationships is poor. }\end{array}$ \\
\hline $\begin{array}{l}\text { The ability to deal } \\
\text { with random events }\end{array}$ & weak & strong \\
\hline
\end{tabular}

\section{Summary and Outlook}

This paper analyzes the design method of adaptive $\mathrm{C} 2$ organization based on granular computing, and raises personal views on the granulation process of platform sets. In the face of the future complex environment of wars, this adaptive design approach can greatly reduce the complexity of problem solving, and improve the efficiency of completing missions. Due to the limited research depth, the paper has not extended or expanded the application of this method in practice. The deficiencies of this method (such as the triggering conditions for the conversion structure, and the selection of the optimal time-domain, etc.) will be analyzed and studied in the next step. The applicable fields of this method will be searched for, and exploration of specific application will be carried out.

\section{References}

[1] B.X. Xiu: Organization structure design method and its robustness and adaptability analysis (Ph.D., National University of Defense Technology, China, 2006), p.25.

[2] L.J. Wan, P.Y. Yao and X. Liang: Electronics Optics \& Control, Vol. 18 (2011) No.11, p. 7.

[3] Y. Yang, B.X. Xiu, T.T. Yang: Command Control \& Simulation, Vol.36 (2014) No.4, p. 1.

[4] B.X. Xiu, W.M. Zhang, Z. Liu: Systems Engineering and Electronics, Vol.29 (2007) No.7, p. 1102

[5] P.Y. Yao, J.Y. Zhang and W. Li: Air Force Engineering University Journal (Natural Edition), Vol.13 (2012) No.2, p. 54.

[6] P.Y. Yao, J.Y. Zhang and L.J. Wan: Air Force Engineering University Journal (Natural Edition), Vol.13 (2012) No.3, p. 54.

[7] D.G. Li, D.Q. Miao and Zhang D. X: Computer science. Vol.32 (2005) No.9, p. 1.

[8] B.X. Xiu, W.M. Zhang and Z. Liu : Progress in Natural Science. Vol.17 (2007) No.5, p.662.

[9] H. Wang, J.H. Wei , C.J. Liu : Journal of Xi'an Jiao-tong University. Vol.47 (2013) No.12, p. 43.

[10] D.S. Yang, X.H. Peng, W.M. Zhang: Fire Control \& Command Control. Vol.31 (2006) No.3, p. 9. 